\title{
Energy Efficient Index for Querying Location-Dependent Data in Mobile Broadcast Environments*
}

\author{
Jianliang $\mathrm{Xu}^{\dagger}$ Baibua Zheng \\ HK Univ. of Sci. \& Tech. \\ Clear Water Bay, HK \\ $\{$ xujl,baihua\}@cs.ust.hk
}

\author{
Wang-Chien Lee \\ Penn State University \\ University Park, PA 16802 \\ wlee@cse.psu.edu
}

\author{
Dik Lun Lee \\ HK Univ. of Sci. \& Tech. \\ Clear Water Bay, HK \\ dlee@cs.ust.hk
}

\begin{abstract}
We are witnessing in recent years growing interest for location-dependent information services among mobile users. This paper examines the issue of processing locationdependent queries in a mobile broadcast environment. Different from a traditional environment, mobile users are concerned with not only access latencies but also power conservation. The planar point location algorithms and conventional spatial index structures are shown inefficient. In this paper, we propose a new index data structure, called $\mathrm{D}$ tree, for querying location-dependent data in mobile broadcast environments. The basic idea is to index data regions based on the divisions between them. We describe how to construct the binary D-tree index, how to process locationdependent queries based on this index structure, and how to page the D-tree to fit the packet capacity. The performance of the D-tree is evaluated using both synthetic and real datasets. Experimental results show that the proposed $D$-tree provides a much better overall performance than the well-known existing schemes such as the $R^{*}$-tree.
\end{abstract}

\section{Introduction}

We are witnessing in recent years growing interest for location-dependent information services (LDISs) among mobile users thanks to the rapid technological development in high-speed wireless networks, personal portable devices, and location identification techniques [7, 20, 23]. Examples of mobile LDISs include location-dependent information access (e.g., traffic reports and attractions) and nearest neighbor queries (e.g., finding the nearest restaurant).

* This work was supported in part by grants from the Research Grant Council of Hong Kong (Grant numbers HKUST6225/02E and AoE/E01/99).

$\dagger$ The author is now with Hong Kong Baptist University, Kowloon Tong, Hong Kong.
Wireless broadcasting is an attractive approach for data dissemination in a mobile environment. Disseminating data through a broadcast channel allows simultaneous access by an arbitrary number of mobile users and thus allows efficient usage of scarce bandwidth. Owing to this scalability feature, the wireless broadcast channel has been considered an alternative storage media (called "air storage") of the traditional hard disks $[1,15]$. Many studies addressing various problems in mobile broadcast environments, such as scheduling and bandwidth allocation, have appeared in the literature $[1,12,15,22]$. It is expected that in the near future a number of mobile LDISs (e.g., region-wide traffic reports and tourism information) will utilize broadcast for the dissemination of information to the rapidly increasing population of mobile users. This paper investigates the issue of querying location-dependent data in mobile broadcast environments.

In wireless broadcast, one critical issue is to conserve battery power, which is a scarce resource for mobile clients. Without any auxiliary information on the broadcast channel, a client may have to access all objects in a broadcast cycle in order to retrieve the desired data. This requires the client to listen to the broadcast channel all the time, which is power inefficient. Air indexing techniques address this issue by pre-computing some index information and interleaving it with the data on the broadcast channel [15]. By first accessing the broadcast index, the mobile client is able to predict the arrival time of the desired data. Thus, it can stay in the power saving mode most of the time and tune into the broadcast channel only when the requested data arrives. The drawback of this solution is that broadcast cycles are lengthened due to additional index information. As such, there is a trade-off between access latency and tuning time. Three criteria have been used to evaluate the performance of air indexing techniques [13, 15]:

- Access Latency: the period of time elapsed from the moment a mobile client issues a query to the moment when the requested data is received by the client. 
- Tuning Time: the period of time spent by a mobile client staying active in order to obtain the requested data.

- Indexing Efficiency: the ratio of the tuning time saved against the non-indexing scheme to the index overhead.

While access latency measures the overhead of an index structure and the efficiency of data and index organization on the broadcast channel, tuning time is frequently used to estimate the power consumption by a mobile client since sending/receiving data is power dominant in a mobile environment [17]. ${ }^{1}$ Indexing efficiency, which correlates access latency and tuning time, is used to evaluate the efficiency of indexing techniques in terms of minimizing tuning time while maintaining acceptable access latency overhead.

Several indexing techniques dedicated to the wireless broadcast channel, i.e., the hashing indexing [14], the signature approach [18], and the hybrid approach [13], have been introduced in the literature. Moreover, imbalanced indexing for skewed data accesses [6] and indexing for multiattribute queries [12] have also been investigated. However, these studies concentrated on one-dimensional indexes for equality-based queries, and thus are inapplicable to location-dependent query processing where point queries are involved. Imielinski et al. investigated the issue of interleaving data and index on the linear wireless channel such that the tuning time is nearly optimized while maintaining the access latency as short as possible $[14,15]$. Recently, there is a study [11] that discussed query processing for spatial objects over the broadcast channel. However, its main focus was on how to utilize the limited client cache to reduce the tuning time when traversing spatial index trees. To the best of our knowledge, the issue of querying locationdependent data in a mobile broadcast environment has not been addressed before.

In this paper, we are interested in exploring efficient index structures for broadcasting location-dependent data on air. We first review some existing index structures. Through an illustration with some simple examples, we show their limitations for querying location-dependent data in mobile broadcast environments. Next, we present a new index data structure, called $D$-tree. The basic idea is to index data regions (i.e., valid scopes of data instances, formally defined in Section 2) based on the divisions between them. We describe how to construct the binary D-tree index, how to process location-dependent queries based on this index structure, and how to page the D-tree to fit the packet capacity. The performance of the D-tree is evaluated using both synthetic and real datasets. Experimental results show that the proposed D-tree provides a much better overall performance than the well-known schemes such as the $\mathrm{R}^{*}$-tree.

\footnotetext{
${ }^{1}$ For systems in which mobile clients are charged on a per-bit basis, tuning time can also be used to measure the access cost.
}

The rest of this paper is organized as follows. Section 2 gives the background on the information system model and the index broadcast model. In Section 3, we review some existing index structures that can be used for querying location-dependent data. Section 4 presents the proposed D-tree index structure along with the partition, query processing, and paging algorithms. The performance evaluation is presented in Section 5. Finally, Section 6 concludes this paper.

\section{Preliminaries}

This section provides some background on locationdependent services and information broadcast systems. It is assumed that location-dependent services are provided to mobile clients by an information broadcast system. We refer to the geographical area covered by the system as the service area, denoted by $\mathcal{A}$. Mobile clients are assumed to be able to identify their locations using such systems as the global positioning system (GPS). A mobile client can move freely from one location to another within the service area and make queries ubiquitously with its current location or a future location to the broadcast system.

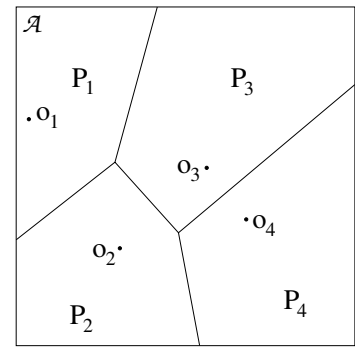

\section{Figure 1. Running Example}

A data type denotes the type of location-dependent information service (e.g., traffic report or nearest restaurant). In this paper, we assume that queries are specified on one data type. The dataset is a collection of data instances that return the answer to a query according to the location it is bound. A data instance has a certain valid scope within which this instance is the only correct answer. For example, in Figure 1, we have four cities, $o_{1}, o_{2}, o_{3}$, and $o_{4}$, and their respective boundaries, $P_{1}, P_{2}, P_{3}$, and $P_{4}$. Given any point in, say, $P_{3}, o_{3}$ is always the city to which the point belongs. This example will be used throughout this paper as the running example for different index structures.

Given a set of data instances and their valid scopes, the problem of querying location-dependent data is, given a query location, how to efficiently determine which data instance to return. In this paper, we study this problem in a two-dimensional space, which is enough for most LDISs. To formulate the problem, we first introduce the concept of data region. A data region is assumed to take the shape of a 
polygon.

Definition $1 A$ data region, $P_{i}$, is spatial representation of the valid scope for a data instance, and one data region corresponds to one data instance, such that $\cup_{i=1}^{N} P_{i}=\mathcal{A}$, and $P_{i} \cap P_{j}=\phi, \forall 1 \llbracket \underline{\underline{\Sigma}} N \quad$ and $i \neq j$, where $\mathcal{A}$ is the service area and $N$ is the number of data instances for one data type.

As can be seen, one salient feature for data regions is that they are connected and adjacent to each other. This study aims at investigating efficient index structures to support location-dependent query processing on a wireless broadcast channel: given data regions $P_{1}, P_{2}, \cdots, P_{N}$, how can all the regions be indexed such that point queries can be processed efficiently in terms of access latency and tuning time?

To interleave data and index on the wireless channel, the $(1, m)$ technique [15] is employed (see Figure 2). That is, the index is broadcast preceding every $\frac{1}{m}$ fraction of the broadcast cycle. As in [15], to reduce the tuning time, each index segment (except for the root) and each data segment contain a pointer pointing to the root of the next index. The access protocol for querying location-dependent data involves the following steps:

- Initial probe: The client tunes into the broadcast channel and determines when the next index will be broadcast. It then turns into the power saving mode until the next index arrives.

- Index search: The client searches the index. It follows a sequence of pointers (i.e., selectively tunes into the broadcast index) to locate the data region containing the query point and find out when to tune into the broadcast channel to get the desired data. It waits for the arrival of the data in the power saving mode.

- Data retrieval: The client tunes into the channel when the desired data arrives and downloads the data.

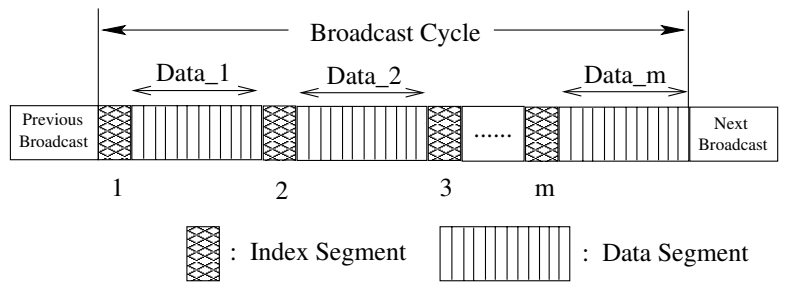

\section{Figure 2. Data and Index Organization on the Broadcast Channel}

In wireless communications, a bit stream is normally delivered in the unit of packet (or frame) for such purposes as error-detecting, error-correction, and synchronization [15]. For example, in the GPRS network a packet can contain the data up to 1600 bytes [5]. As a result, data is accessed by clients in the unit of packet. Thus, the tuning time for an index structure is measured in terms of the number of packet accesses $[12,15]$.

\section{Review of Existing Approaches}

In general, indexes based on simple shapes perform efficiently. Thus, the actual shape of a spatial object is often abstracted before being inserted to the index. To serve this purpose, there are two categories of techniques: object decomposition and object approximation [4, 9]. In object decomposition, the shape of each object is represented as the geometric union of simpler shapes such as triangles and trapezoids. In object approximation, regular shapes such as bounding rectangles or spheres are used to approximate spatial objects. In the following subsections, we briefly introduce several typical solutions and analyze their limitations when they are applied to location-dependent queries in a mobile broadcast environment.

\subsection{Object Decomposition}

The object decomposition technique has been developed for the planar point location problem [3]: given a polygonal subdivision of the plane ( $n$ vertices and $m$ segments) and a query point $p$, how can we efficiently determine which face of the subdivision contains $p$ ? This is similar to our problem. However, we are concerned with not only search efficiency (which contributes to the tuning time) but also access latency (which is attributed to the index storage size). As a result, the point location algorithms perform poorly overall as we will show in the performance evaluation later in this paper. There is a long stream of research on the point location problem. Kirkpatrick's algorithm [16] and the trapezoidal map approach [3] are two typical solutions. The former achieves an $\mathrm{O}(\log n)$ search time and an $\mathrm{O}(n)$ space, while the latter is a randomized algorithm and has an expected search time of $\mathrm{O}(\log m)$ and an expected space of $\mathrm{O}(m)$. However, the constant factors in these worst performance measures are large, especially for the space measure [3].

In Kirkpatrick's algorithm, the original subdivision is first triangulated. Then, we recursively remove some vertices, along with all the edges adjacent to them, and retriangulate the new subdivision. This operation is continued until the number of triangles contained in the space is smaller than some predefined threshold $\left(T_{m i n}\right)$. Figures 3(a), 3(b), and 3(c) show the triangulation processing for our running example, where $T_{\min }$ is set to five. From 3 (a) to 3(b), vertices $v_{3}, v_{5}$, and $v_{6}$ are removed; and from 3(b) to 3(c), vertex $v_{4}$ is removed. A hierarchical index tree, as shown in Figure 3(d), is built upon the triangles generated in the course of recursive triangulation. Given 


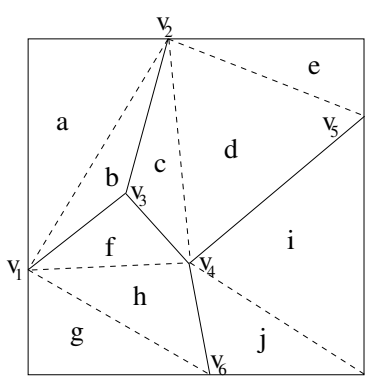

(a) Triangulation of the original subdivision

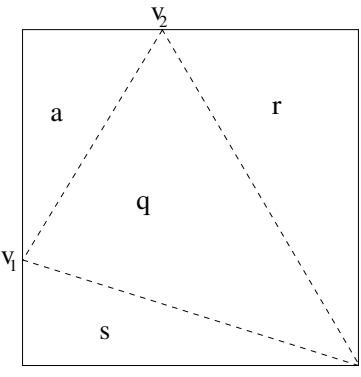

(c) Re-triangulation after $v_{4}$ is also removed

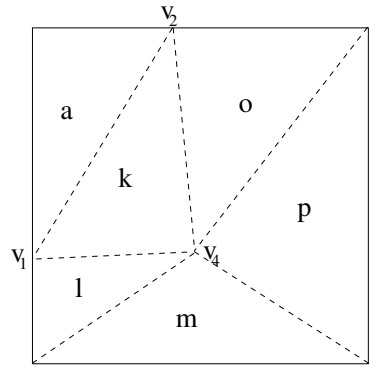

(b) Re-triangulation after $v_{3}, v_{5}$, and $v_{6}$ are removed

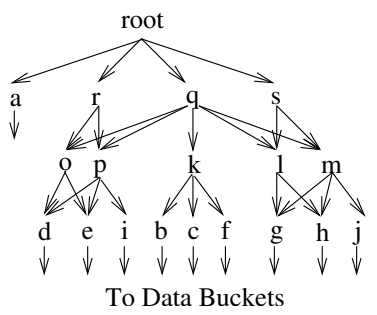

(d) The Index structure
Figure 3. Index Construction Using Kirk-
patrick's Algorithm (the Trian-Tree)

a query point, the probe begins at the root. It checks each child sequentially until a triangle containing the query point is found. Then, the search continues from that node all the way down to a leaf node. In this paper, we call the index structure built by Kirkpatrick's algorithm the trian-tree.

In the trapezoidal map approach, the planar subdivision is viewed as a collection of line segments. The index structure is built when the line segments are inserted one by one into the subdivision. From each new vertex created by the insertion of a line segment, we draw two vertical extension lines, one going upwards and the other going downwards. The extension does not stop until it meets a line segment that has been previously inserted. Eventually, the original subdivision is decomposed into a set of trapezoids. Obviously, the insertion order of line segments influences the index structure. A randomized incremental approach is employed in our experiments [3]. Figure 4(a) shows the final trapezoidal map of our example, where line segments are inserted in the order of $s_{1}, s_{2}, s_{3}, s_{4}$, and $s_{5}$. The corresponding index structure is shown in Figure 4(b). There are two kinds of nodes in the index structure: one is $x$-node (represented by a circle) recording the $\mathrm{x}$-coordinate of a vertex; the other is $y$-node (represented by a hexagon) recording a line segment. Given a query point $p$, the search process begins at the root and terminates when a leaf node is met. At an $\mathrm{x}$-node, we evaluate whether $p$ lies to the left or to

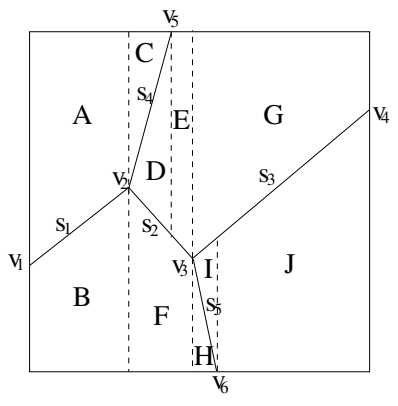

(a) Final trapezoidal map

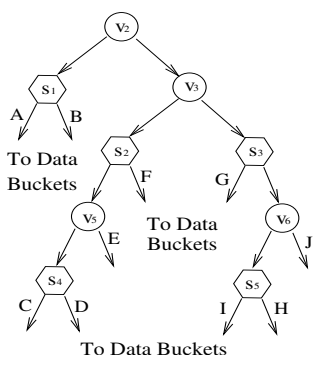

(b) Index structure

\section{Figure 4. Index Construction Using Trape- zoidal Map (the Trap-Tree)}

the right of the vertical line that goes through the stored $\mathrm{x}$ coordinate. At a y-node, we evaluate whether $p$ lies above or below the stored line segment. We call the index structure built using the trapezoidal map approach the trap-tree in this paper.

For both the trian-tree and the trap-tree, an intuition is that their index sizes are probably large. For our example with only four regions and six vertices, the index trees have about ten nodes. This will significantly increase the broadcast cycle and result in a long access latency in a wireless broadcast environment.

\subsection{Object Approximation}

The object approximation technique has been commonly employed for disk indexing in the spatial database field [9]. The problem is that given a very large amount of spatial objects, efficient disk indexes need to be created to support various spatial operations. ${ }^{2}$ Since the index data on a disk is accessed in the unit of page and the disk access time is pre-dominant in access latency, the objective of an index structure is often to minimize the number of page accesses. This seems similar to our case that we have to access the index on air in the unit of packet and we want to optimize the number of packet accesses. However, it is worth noting that the motives are different: disk indexing is required only for large databases, whereas packet-based data access is a "physical" requirement for wireless communications. Hence, in our case minimizing the amount of packet accesses is required for databases of any size. Moreover, as we will illustrate in a moment, the nature of our problem renders the approximation-based spatial index structures inefficient.

One of the most classical spatial index structures is the $\mathrm{R}$-tree [10]. There are also some variants such as the $\mathrm{R}^{+}$tree [21] and the $\mathrm{R}^{*}$-tree [2]. The basic idea is to use a

\footnotetext{
${ }^{2}$ Due to a large amount of objects, the main memory may not be able to accommodate the index structure so that it has to be stored on a disk.
} 


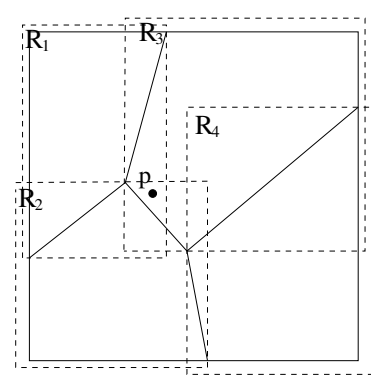

(a) MBRs of data regions

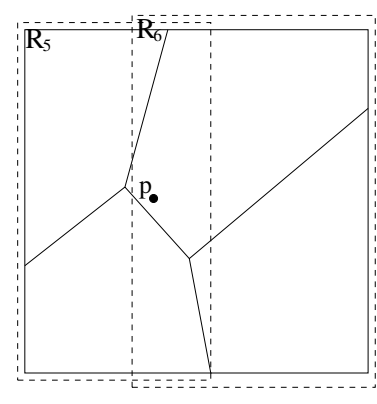

(b) MBRs in the root

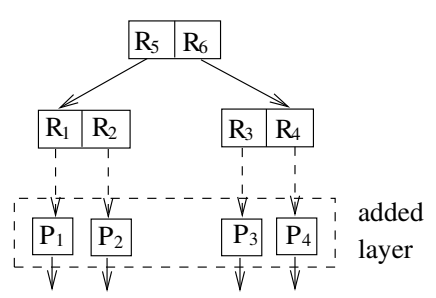

To Data Buckets

(c) Index structure

\section{Figure 5. Index Construction Using the $\mathbf{R}^{*}$ - tree}

minimal bounding rectangle (MBR) to approximate a spatial object, and then establish the index based on a sequence of MBRs. Each node in the index tree contains a number of entries according to the page capacity. An entry in an internal node contains a child-pointer pointing to a lower level node in the tree and a bounding rectangle covering all the rectangles in the lower nodes in the subtree. In a leaf node, an entry consists of a pointer pointing to the data and a bounding rectangle which bounds its data objects. Variants of the R-tree differ from one another in the criteria used to insert an object and to split an overflowing node. Extensive experiments conducted in [2] showed that the $\mathrm{R}^{*}$-tree gained a superior performance for different types of queries and operations. Figure 5(c) shows the structure of the $\mathrm{R}^{*}$ tree (excluding the added layer) for our example, where the corresponding MBRs are shown in Figures 5(a) and 5(b). In the performance evaluation, the $\mathrm{R}^{*}$-tree is used as the representative of the object approximation technique.

Given a query point $p$, the search algorithm descends the tree from the root. The algorithm recursively traverses down the subtrees of bounding rectangles that contain $p$. When a leaf node is reached, bounding rectangles are tested and their objects are fetched to verify whether they contain $p$. When applying the $\mathrm{R}^{*}$-tree to the wireless broadcast scenario, to save the tuning time, we modify the tree structure slightly: one layer is added to the $\mathrm{R}^{*}$-tree at the bottom as shown in Figure 5(c). This layer consists of the actual shapes of data regions so that in the containment test a costly access of actual data is avoided.
As can be seen, the problem with the R-type tree is that if a point is covered by two or more sibling MBRs it may need to explore several subtrees before the wanted object can be located. This will increase the search time and hence the tuning time. Unfortunately, in our scenario, all data regions are adjacent to each other, and, as such, their MBRs will overlap. The nature of this problem renders the approximation-based spatial index structures inefficient. As an example, suppose the query point is $p$ in Figure 5(a) and 5(b). The search first reaches the leaf node $R_{1}$ through the root and $R_{5}$. Since it is outside $P_{1}$ pointed by $R_{1}$, the search backtracks to $R_{2}$. Likewise, it is outside of $P_{2}$ and next the search backtracks to $R_{6}$. Finally, it obtains the correct answer in node $R_{3}$. Thus, we need to access a total of six nodes (i.e., the root, $R_{5}, R_{1}, R_{2}, R_{6}$, and $R_{3}$ ) before we know it is contained in $P_{3}$.

\section{The D-tree Index Structure}

This section describes the index data structure of the proposed D-tree. In the following, we first present the overall idea of the D-tree in Section 4.1. The partition algorithm is described in Section 4.2. Section 4.3 provides the algorithm for location-dependent query processing based on the D-tree index structure. Finally, Section 4.4 explains how to page the binary D-tree to fit the packet capacity.

\subsection{An Overall Picture}

As discussed in the last section, the object decomposition and approximation approaches suffer from a long access latency and/or a long tuning time. In the meantime, we observe that the actual shapes of data regions are contained, either explicitly or implicitly, in the index structures of both approaches. In object decomposition, the shape of each region is embedded in the index structure, while in object approximation, it is approximated by an MBR with the exact shape encoded in an additional layer of nodes, as shown in Figure 5(c). Based on this observation, we propose a new data structure, called $D$-tree, to index data regions directly based on the divisions between them. This new index structure neither decomposes nor approximates data regions. In the following, we illustrate the overall idea.

The D-tree is a binary height-balanced tree similar to the kd-tree [9]. However, while the kd-tree is built based on hyperplanes, the D-tree is built based on the divisions between data regions. We recursively partition a space consisting of a set of data regions into two complementary subspaces containing about the same number of regions until each subspace has one region only. The partition between two subspaces is represented by one or more polylines. The overall orientation of the partition, hereafter referred 
to as partition dimension, can be either $x$-dimensional or $y$ dimensional, which is obtained, respectively, by sorting the data regions based on their lowest/uppermost y-coordinates, or leftmost/rightmost x-coordinates (see Section 4.2). Figure 6(a) shows the partitions for our running example. The polyline $p l\left(v_{2}, y_{3}, v_{4}, v_{6}\right)$ partitions the original space into $P_{5}$ and $P_{6}$, and $p l\left(v_{1}, v_{3}\right)$ and $p l\left(v_{4}, v_{5}\right)$ further partition $P_{5}$ into $P_{1}$ and $P_{2}$, and $P_{6}$ into $P_{3}$ and $P_{4}$, respectively. The first polyline is $y$-dimensional and the remaining two are $x$-dimensional. The algorithm of finding the partition for a space will be described in Section 4.2.

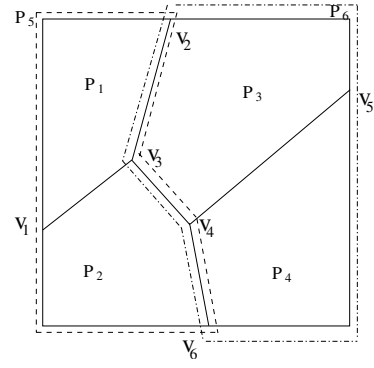

(a) Divisions for the example

Figure 6. Index Construction Using the D-tree

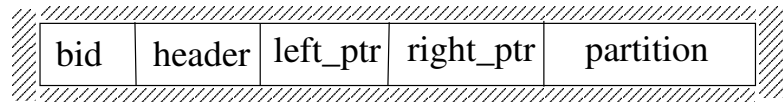

Figure 7. Data Structure of the D-tree Node

\begin{tabular}{|l||l|}
\hline Attribute & Description \\
\hline \hline bid & the unique id for each node \\
\hline header & $\begin{array}{l}\text { include flag indicating if the node size }>\text { one } \\
\text { packet, style \& size of the partition }\end{array}$ \\
\hline left_ptr & $\begin{array}{l}\text { the type (data or node pointer) and the } \\
\text { offset to the beginning of the left child }\end{array}$ \\
\hline right_ptr & $\begin{array}{l}\text { the type (data or node pointer) and the } \\
\text { offset to the beginning of the right child }\end{array}$ \\
\hline partition & $\begin{array}{l}\text { a sequence of coordinates that represent } \\
\text { the partition }\end{array}$ \\
\hline
\end{tabular}

Table 1. Illustration of the Attributes in a Dtree Node

The data structure of a D-tree node is illustrated in Figure 7. The meaning of each attribute is summarized in Table 1 . In the D-tree, an internal node contains the partition that divides the current space into two complementary subspaces, a left (right) pointer pointing to the node containing the data regions that lie in the lefthand or upper (righthand or lower) subspace, and some control parameters including bid and header. A leaf node contains the partition of two data regions, the pointers pointing to the data buckets corresponding to the regions, and the control parameters as well. Thus, a spatial data region is inferred by the partitions when following the path from the root towards a leaf node. Note that in the data structure of Figure 7, we place the pointers before the partition on purpose. We will explain this in Section 4.4. For the moment, there is no difference for where the pointers are placed. The binary D-tree satisfies the following four properties (the correctness was proved in [24]).

1. Every node has exactly two children.

2. All objects in the left subtree of a node are in the lefthand or upper subspace of the partition, and all objects in the right subtree are in the righthand or lower subspace.

3. The tree is height-balanced, i.e., the levels of the leaves differ by at most one.

4. The search time for a point query is $\Theta(\log N)$ in terms of the number of nodes visited.

The D-tree index structure for the running example is depicted in Figure 6(b), where the header attribute is simplified and only contains the partition dimension. Compared to the trian-tree in Figure 3 and the trap-tree in Figure 4, the size of the $\mathrm{D}$-tree is much smaller. Compared to the $\mathrm{R}^{*}$-tree in Figure 5, the search time for the D-tree is expected to be shorter since it searches only two nodes for any query point.

\subsection{Partition Algorithm}

Finding a good partition for a space is crucial to the efficiency of the D-tree. This subsection describes the proposed space partition algorithm. There are many ways of dividing one space into two complementary subspaces that contain almost the same number of data regions. For example, we can sort the regions according to their rightmost $\mathrm{x}$-coordinates, and identify the space that encloses the first $N / 2$ regions as one subspace and the rest as the other. Alternatively, we can sort the regions according to their lowest $\mathrm{y}$-coordinates, leftmost $\mathrm{x}$-coordinates, or uppermost $\mathrm{y}$ coordinates, and perform the subspace identification in a similar fashion. Moreover, if $N$ is odd, we may identify the first $(N+1) / 2$ or $(N-1) / 2$ regions as one subspace. Consequently, four partition styles are evaluated when $N$ is even and eight when $N$ is odd.

For each partition style, the size of the partition is measured in terms of the number of coordinates that represent the partition. In selecting among different partition styles, we choose the one with the smallest partition size. If they are equal, for tie breaking, we define inter-prob of two subspaces as the probability of a query being issued from their interlocking part, i.e., for a $y$-dimensional ( $x$-dimensional) partition, the area between the rightmost x-coordinate (lowest $y$-coordinate) of the lefthand (upper) subspace and the 


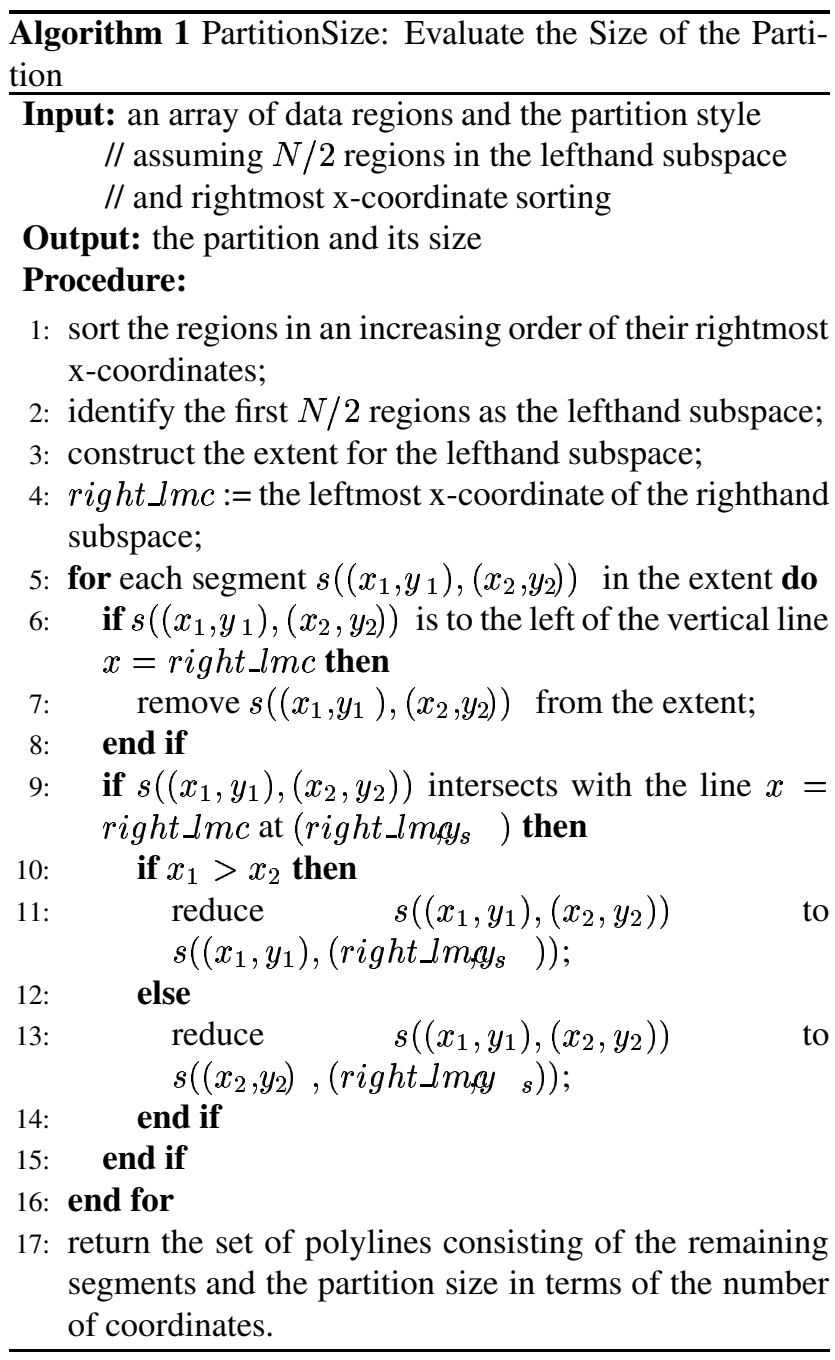

leftmost x-coordinate (uppermost y-coordinate) of the righthand (lower) subspace (e.g., $D_{2}$ in Figure 8(a)). Ties are broken by favoring the one with the lowest inter-prob. The reason will become clear when the reader proceeds to the next two subsections and we will mention it again later on in Section 4.4.

We now describe the algorithm PartitionSte (Algorithm 1) that takes an array of data regions and the partition style as the input and evaluates the partition size with the input style. To simplify the illustration, the algorithm is presented for the style in which the sorting is based on the regions' rightmost $\mathrm{x}$-coordinates, and $N / 2$ regions are given to the lefthand subspace. It is obvious to extend it to other partition styles. The algorithm consists of two phases. In the first phase (lines 1-3), we identify the lefthand subspace and construct the extent for this subspace. This is straightforward. However, it is worth noting that the extent of a subspace could consist of one or more closed polygons. ${ }^{3}$ In

\footnotetext{
${ }^{3}$ For example, in Figure $6(\mathrm{a})$, when the partition $p l\left(v_{2}, v_{3}, v_{4}, v_{6}\right)$ has
}

the second phase (lines 4-16), we prune some unnecessary segments in the extent such that the remaining segments are sufficient to guide a query point to the appropriate subspace. Thus, we prune the segments that are to the left of the vertical line that goes through the leftmost $\mathrm{x}$-coordinate of the righthand subspace, right $\_m c$ (lines 6-8). In addition, we truncate the remaining segments by right $\operatorname{Imc}$ (lines 9-15). Note that this operation does not change the partition size, but it identifies right_lmc in the partition, which is useful in paging (see Section 4.4). Figure 8(a) gives an example where the extent of the lefthand subspace is the union of the dash-dot line and the solid line. The output partition of Algorithm 1 for this example would be the solid line. The complexity of Algorithm 1 is $\mathrm{O}(N \log N+M)$, where $N$ is the number of regions and $M$ is the number of line segments. Therefore, the complexity of the recursive partition procedure for the original space is $\mathrm{O}\left(N \log ^{2} N+M \log N\right)$.

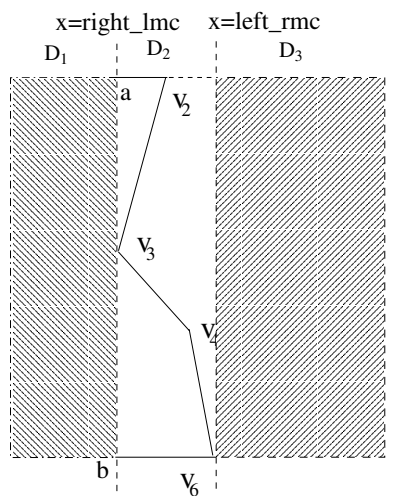

(a) Partition

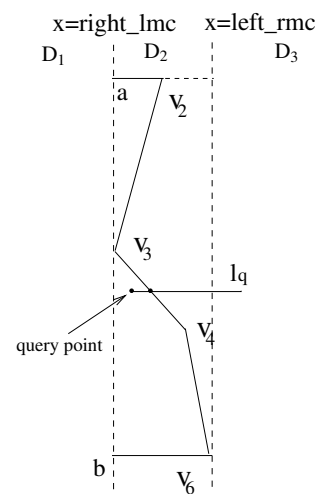

(b) Query processing

\section{Figure 8. Examples for the Partition and Query Processing Algorithms}

\subsection{Query Processing Algorithm}

This subsection presents the algorithm for processing location-dependent point queries based on the D-tree index structure. As we discussed in Section 2, the D-tree records data regions to facilitate point queries. Thus, given a query point, the query processing problem becomes equivalent to the problem of searching in the D-tree for the data region containing the query point. The search algorithm works as follows. It starts from the root and recursively follows either the left pointer or the right pointer according to the query point and the partition until a leaf node is reached. Since the D-tree is a binary height-balanced tree and there is no spatial overlapping among sibling nodes, the search time is $\Theta(\log N)$ in terms of the number of nodes visited. The procedure is described in Algorithm 2, where the partition

one or more vertices (say, $v_{3}$ and/or $v_{4}$ ) touching the left edge of the space, the left subspace will consist of more than one closed polygon. 


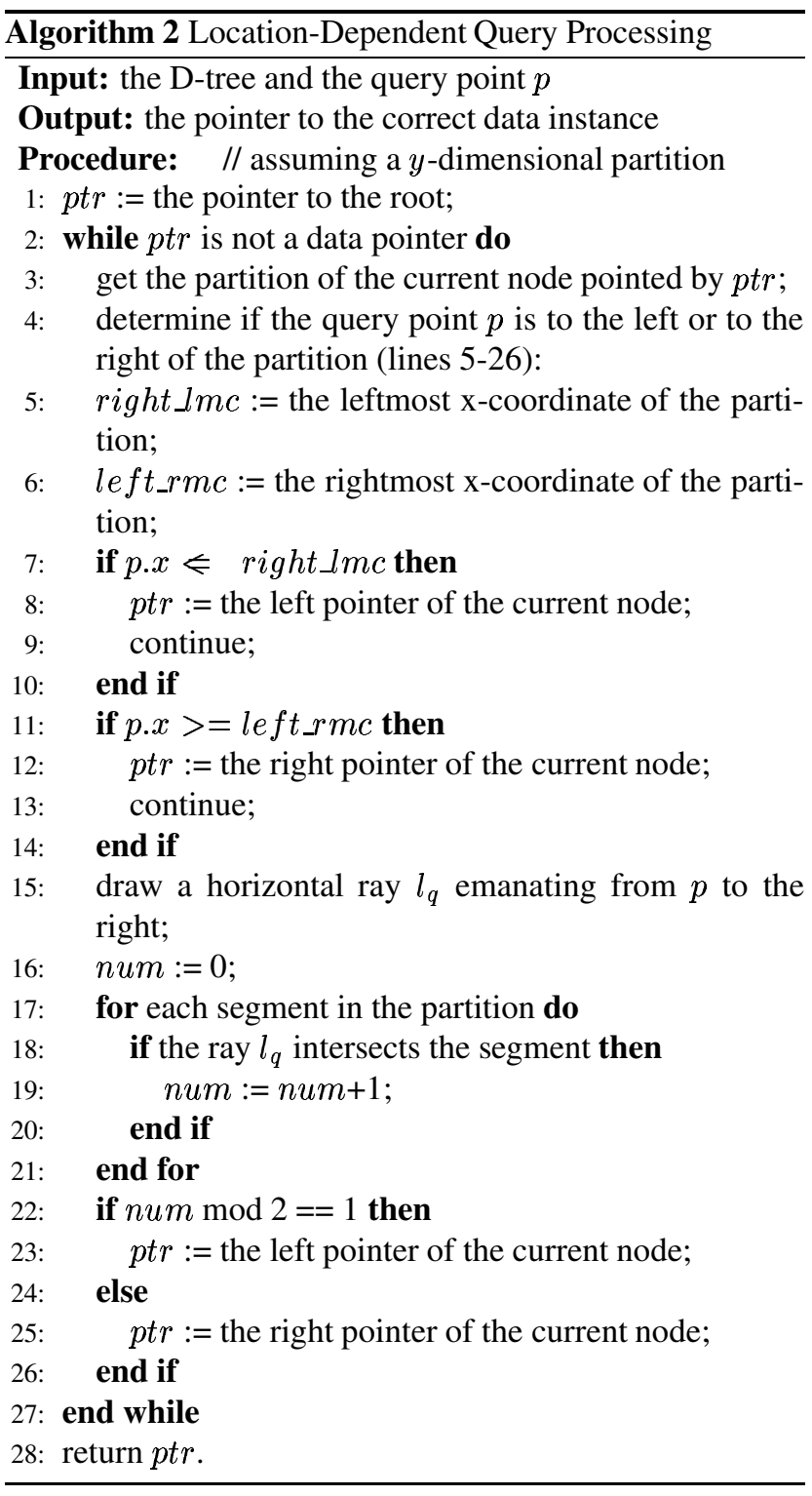

style is assumed to be a $y$-dimensional partition. It is trivial to extend the algorithm to the $x$-dimensional partition style.

In the algorithm, the key issue is to determine whether a given query point $p$ is located to the left or to the right of the partition. As shown in Figure 8(a), we can see that after a partition, a space is divided into three parts: $D_{1}, D_{2}$, and $D_{3}$, where $D_{1}$ is bound by the leftmost x-coordinate of the righthand subspace (i.e., right $l m c$ ) and $D_{3}$ is by the rightmost $\mathrm{x}$-coordinate of the lefthand subspace (i.e., left_rmc). If $p$ falls in $D_{1}$ (i.e., $p . x \leqslant$ right $l m c$ ), it goes to the lefthand subspace (lines 7-10). If $p$ falls in $D_{3}$ (i.e., $p . x>=$ left_rmc), it goes to the righthand subspace (lines 11-14). Otherwise, $p$ falls in $D_{2}$ and it has to be determined by examining the partition since $D_{2}$ is shared by both subspaces (lines 15-26). This operation is illustrated by an example. In Figure 8(b), Suppose that the solid line is the partition and that the query point is $p$. Consider a horizontal ray $l_{q}$ emanating from $p$ to the right. If the number of times that this ray $l_{q}$ intersects the line segments making up the partition is odd, then $p$ is to left of the partition; otherwise if the number is even, then $p$ lies to the right of the partition. In Figure 8(b), since the number turns out to be one, we know the query point is in the lefthand subspace.

\subsection{Paging the D-tree}

As discussed in Section 2, wireless data is accessed in the unit of packet. Thus, it needs to allocate the nodes of the binary D-tree into packets of fixed size. In this paper, we employ a top-down approach to carry out packet allocation [19]. The algorithm works as follows. The D-tree is traversed in a breadth-first order. When inserting a new node, if the inclusion of the new node in the packet where the parent node is allocated does not exceed the packet capacity, the new node is allocated space in this packet. Otherwise, a new packet is created and the new node is allocated to the beginning of this packet. We do not split a tree node unless it it is larger than the packet capacity since splitting a small node will result in a two-packet access, instead of one if the node is not split. Finally, to save storage we merge some partial packets at the leaf level in a greedy way. The pseudo code of the procedure is described in Algorithm 3. This algorithm has a complexity of $\mathrm{O}(N)$. The query processing over the paged D-tree is similar to that over the binary D-tree.

For a large node that occupies more than one packet, the following special arrangement is made. An additional coordinate, $R M C$ (i.e., the rightmost $\mathrm{x}$-coordinate in the partition for a $y$-dimensional partition or the lowest $y$-coordinate for an $x$-dimensional partition), is inserted before the partition, and the partition starts with the point of $L M C$ (i.e., the leftmost coordinate in the partition for a $y$-dimensional partition or the uppermost coordinate for an $x$-dimensional partition). The previous example in Figure 8(a) is used to demonstrate the advantage of this arrangement. For query points falling in $D_{1}$ and $D_{3}$, once knowing $R M C$ and $L M C$, we can detect which pointer to follow in the first packet, thereby preventing further packet accesses and reducing the tuning time. Consequently, we place the pointers before the partition to support this early termination of packet accesses, as discussed in Section 4.1. In addition, it is obvious that for two partition styles of the same size, the lower the inter-prob of two subspaces, the higher the probability of successful early detection of the next pointer. For nodes with sizes larger than the packet capacity, this will result in a shorter index search time. Therefore, in the space partition algorithm (Section 4.2), we break ties by favoring the partition style with a lower inter-prob. 


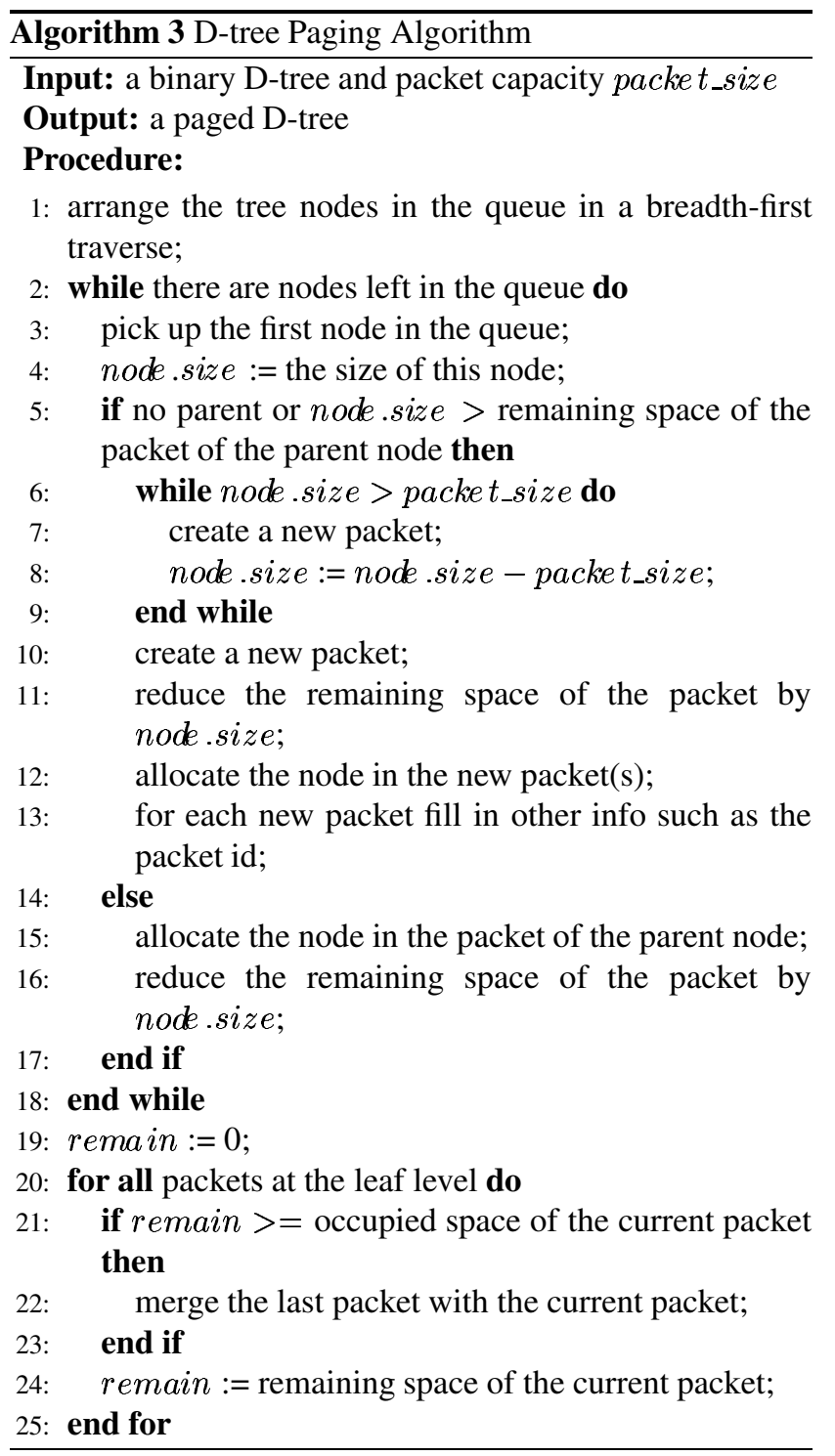

\section{Performance Evaluation}

This section presents the performance evaluation results for the proposed D-tree index structure. Three datasets are used in the experiments (see Figure 9). In the first dataset (UNIFORM), we randomly generate 1000 points in a square Euclidean space. The second set (HOSPITAL) and the third set (PARK) contain the positions of the hospitals and parks in Southern California, which are extracted from the point dataset available from [8]. The valid scopes (or data regions) of the points regarding nearest neighbor search, as shown in the figures, are constructed using the Voronoi Diagram approach [3]. The distributions of the data regions in the latter two datasets are highly clustered. A uniform access distribution over the data regions is assumed in the evaluation.

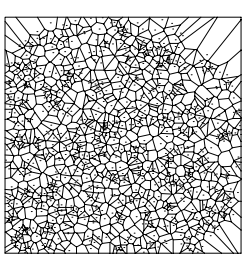

(a) UNIFORM

$(N=1000)$

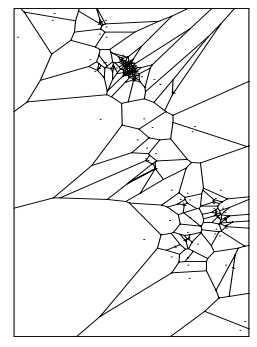

(b) HOSPITAL

$(N=185)$

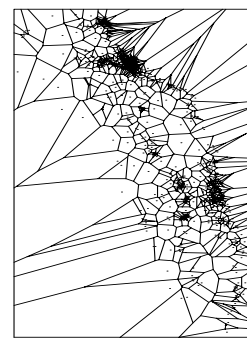

(c) PARK

$(N=1102)$
Figure 9. Datasets under Evaluation

\begin{tabular}{|l||l|l|l|}
\hline Parameter & D-Tree & $\begin{array}{l}\text { Trian-Tree } \\
\text { Trap-Tree }\end{array}$ & R*-Tree \\
\hline \hline bid size & 2 bytes & 2 bytes & 2 bytes \\
\hline header size & 2 bytes & 0 & 0 \\
\hline pointer size (each) & 4 bytes & 4 bytes & 2 bytes \\
\hline coordinate size & \multicolumn{3}{|c|}{ 4 bytes } \\
\hline data instance size & \multicolumn{3}{|c|}{ 1K bytes } \\
\hline packet capacity & \multicolumn{3}{|c|}{64 bytes - 2K bytes } \\
\hline
\end{tabular}

Table 2. System Parameters Setting

The D-tree is compared to the trian-tree, the trap-tree, and the $\mathrm{R}^{*}$-tree index structures. As in the $\mathrm{D}$-tree, the nodes in the trian-tree and the trap-tree do not fit the packet capacity either. To remedy this situation, we page the traptree using the top-down paging approach. For the trian-tree, the nodes are paged in a greedy way as they are traversed in a breadth-first order. This is because in the trian-tree a node may be pointed by more than one parent node (see Figure 3), making the top-down paging approach impractical. For the $\mathrm{R}^{*}$-tree, it is obvious that a better search approach is to examine candidate packets in a depth-first order, such that once a containment test in leaf nodes evaluates to true, the search can be terminated without accessing useless branches. We employ this search method for the $\mathrm{R}^{*}$ tree in the experiments. To reduce storage size, the added layer in the $\mathrm{R}^{*}$-tree is also paged in a greedy manner. The trap-tree and the D-tree are broadcast on the wireless channel in a breadth-first order to facilitate merging of the leaf nodes. The trian-tree is broadcast in a breadth-first order due to the aforementioned reason. The $\mathrm{R}^{*}$-tree is broadcast in a depth-first order to enable backtracking operations.

The system parameters for the evaluation are set as in Table 2. For the trian-tree and the trap-tree, the header size is set to 0 since the size of a triangle or a segment is fixed and there is no need to specify the size of each partition. For the $\mathrm{R}^{*}$-tree, the pointer size is set to 2 bytes since its nodes fit the packet capacity well and a pointer is just the offset to the beginning of the packet containing its child. The header 


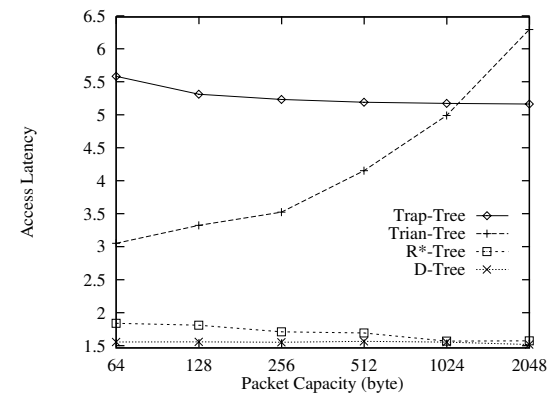

(a) UNIFORM

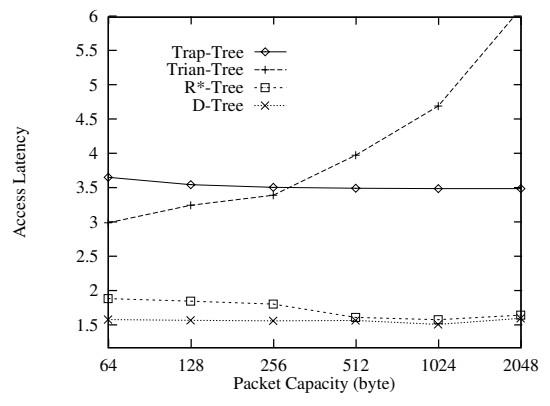

(b) HOSPITAL

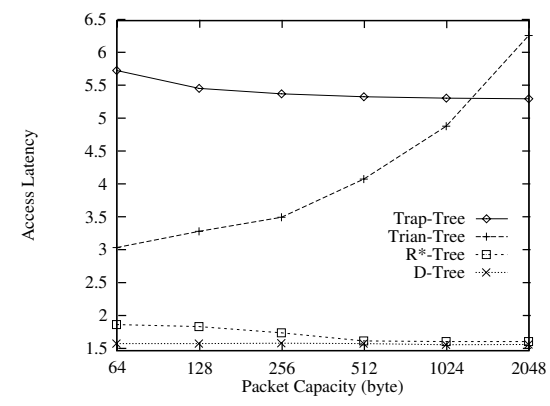

(c) PARK

Figure 10. Performance of Expected Access Latency

info is also unnecessary in the $\mathrm{R}^{*}$-tree.

We assume that flat broadcast is employed for broadcasting data on the wireless channel. The $(1, m)$ interleaving technique [15] is used to interleave the index and the data on the broadcast channel. The optimal value of $m$ depends on the index size. It is calculated for each index structure separately based on the technique presented in [15]. The results are obtained based on 1,000,000 randomly generated queries. In the following three subsections, we present the results in terms of access latency, tuning time, and indexing efficiency, respectively.

\subsection{Access Latency}

This subsection evaluates the access latencies of the various indexes. Figures 10(a), 10(b), and 10(c) show the results as a function of packet capacity for the three datasets, respectively. The latencies in the figures are normalized to the expected access latency without any index (or called the optimal access latency, i.e., half the time needed to broadcast the database). The access latency is affected by the index size. The larger the index size, the longer the access latency. Figure 11 shows the normalized index sizes of the indexes for the PARK dataset. Comparing Figure 11 and Figure 10(c), we can see that the relative performance in index size and access latency is consistent.

Let's compare the performance of different index structures. From Figure 10, the trian-tree and the trap-tree have the worst performance, with an expected latency several times of the optimal latency. This indicates that these two index structures are almost impractical unless they can provide an extremely good tuning time and one is concerned with the tuning time only. As expected, the D-tree gets the access latency no longer than the $\mathrm{R}^{*}$-tree, and is much better than the $\mathrm{R}^{*}$-tree for a small packet capacity. The access latency overhead due to the D-tree indexing is maintained at a similar level for all settings of the packet capacity. It is about $50 \%$ worse than the optimal latency in all the three datasets. We expect that the index overhead at this level is

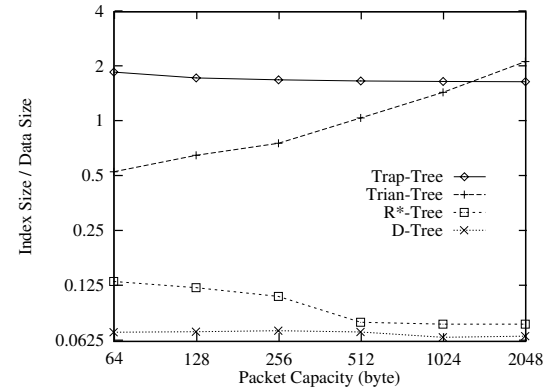

\section{Figure 11. Normalized Index Sizes for the PARK Dataset}

acceptable provided that a good tuning time is achieved.

\subsection{Tuning Time}

This subsection investigates the tuning time for the index structures. To have a close comparison, we measure the tuning time only for the index search step since the tuning time for the steps of initial probe and data retrieval is the same for all the index structures.

As shown in Figure 12, the $\mathrm{R}^{*}$-tree has the worst performance because of a significant degree of overlap among index subspaces, which often carries out a search to access more than one leaf node before the desired pointer can be reached. For all the three datasets, the D-tree gains a much better performance than the trian-tree and the trap-tree when the packet has a capacity larger than 256 bytes. When the packet capacity is smaller than 256 bytes, the D-tree performs slightly worse than the trap-tree. This can be explained as follows. It was observed that, compared with the trap-tree, in the D-tree, the partition size for the nodes at the top levels are a little bit larger. Thus, for a small packet capacity, a top-level node requires more packets to store. As an index search has to access the top-level nodes, this leads to a poorer performance for the D-tree. When the packet capacity increases, the binary D-tree can take advantage of the top-down paging approach and allocate a large number of 


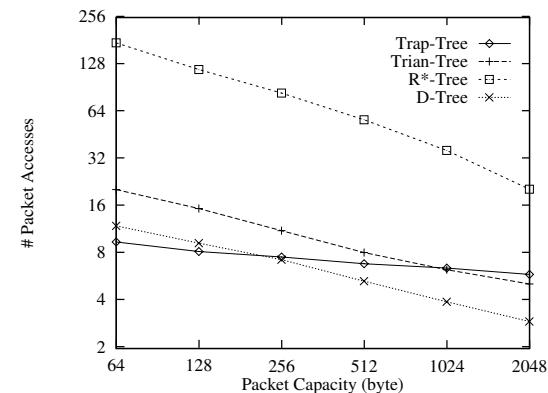

(a) UNIFORM

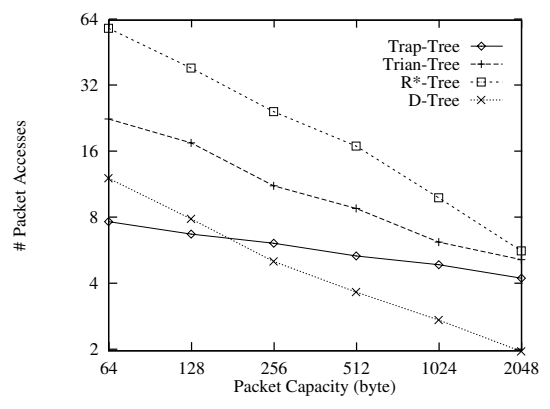

(b) HOSPITAL

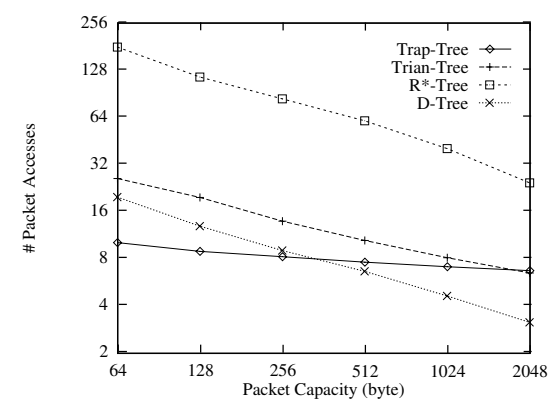

(c) PARK

Figure 12. Peformance of Tuning Time

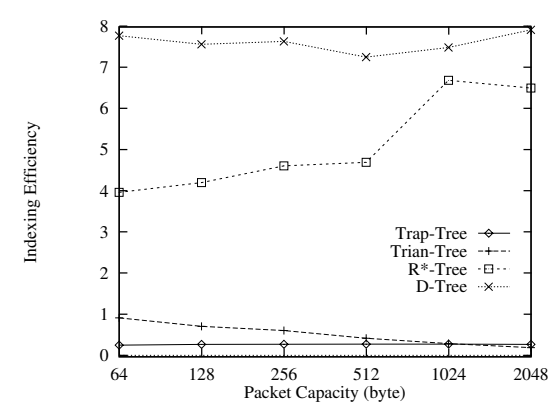

(a) UNIFORM

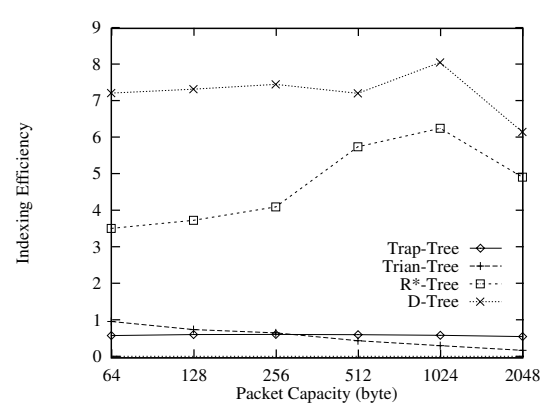

(b) HOSPITAL

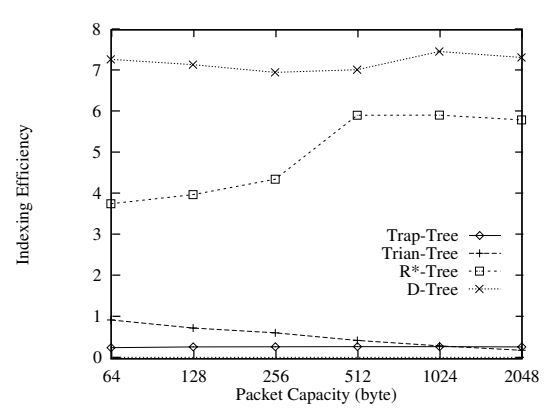

(c) PARK

Figure 13. Performance of Indexing Efficiency

branches to a single packet. This decreases the tree height greatly and hence reduces the search time. However, the trap-tree cannot compress the tree very much because of its large index size (see Figure 11). As a result, the tuning time of the D-tree is only about half that of the trap-tree when the packet has a large capacity.

\subsection{Indexing Effi ciency}

We now evaluate the index structures in terms of indexing efficiency. The larger the indexing efficiency, the more valuable the index. The results for the three datasets are shown in Figures 13(a), 13(b), and 13(c), respectively.

Due to an extremely large index size, the trap-tree has the worst performance, although it can provide a short tuning time for a small packet capacity in Figure 12. With a medium index size, the trian-tree performs better than the trap-tree. However, the performance of these two index structures is far from that of the $\mathrm{R}^{*}$-tree and the $\mathrm{D}$-tree. The proposed $\mathrm{D}$-tree is superior in all cases. This means that the best tradeoff between tuning time and index size is achieved by the D-tree. This is expected since, as shown in the last two subsections, the D-tree can provide a very good tuning time while maintaining the index overhead at a reasonable level.

\section{Conclusion}

While LDISs are becoming increasingly popular among mobile users, data broadcast provides an elegant scalability to an unlimited client population. It is natural to employ data broadcast to disseminate location-dependent data (such as region-wide information) to mobile users. In this paper, we have studied the issue of querying location-dependent data in a mobile broadcast environment.

Through careful analysis of some existing index structures, we found these indexes inefficient for LDISs implemented in a mobile broadcast environment. A new index structure, called D-tree, has been proposed. Different from the existing approaches, the D-tree neither decomposes nor approximates data regions, rather indexes them directly based on the divisions between the regions. The partition algorithm, the query processing algorithm, and the paging algorithm for the D-tree have been described.

We have evaluated the performance of the proposed $\mathrm{D}$ tree thoroughly using both synthetic and real datasets. The following results are obtained. In terms of access latency, the D-tree substantially outperforms the planar point algorithms (i.e., the trian-tree and the trap-tree) and maintains a similar level of index overhead to the $\mathrm{R}^{*}$-tree. In terms of tuning time, the $\mathrm{D}$-tree index shows a much better perfor- 
mance than the trap-tree for a large packet capacity, and the $\mathrm{R}^{*}$-tree and the trian-tree in all cases. It performs slightly worse than the trap-tree only when the packet capacity is very small. As a result, the D-tree provides the best overall performance in terms of indexing efficiency. As such, the D-tree index is recommended for practical use in querying location-dependent data in mobile broadcast environments.

\section{References}

[1] S. Acharya, R. Alonso, M. Franklin, and S. Zdonik. Broadcast disks: Data management for asymmetric communications environments. In Proc. ACM SIGMOD, pages 199-210, May 1995.

[2] N. Beckmann and H.-P. Kriegel. The $\mathrm{R}^{*}$-tree: An efficient and robust access method for points and rectangles. In Proc. ACM SIGMOD, pages 322-331, 1990.

[3] M. Berg, M. Kreveld, M. Overmars, and O. Schwarzkopf. Computational Geometry. New York: Springer-Verlag, 1997.

[4] E. Bertino, B. C. Ooi, R. Sacks-Davis, K. L. Tan, J. Zobel, B. Shilovsky, and B. Catania. Indexing techniques for advanced database systems. Boston: Kluwer Academic, 1997.

[5] J. Cai and D. J. Goodman. General packet radio service in GSM. IEEE Communications Magazine, 35(10):122-131, Oct. 1997.

[6] M.-S. Chen, P. S. Yu, and K.-L. Wu. Indexed sequential data broadcasting in wireless mobile computing. In Proc. IEEE ICDCS, pages 124-131, May 1997.

[7] K. Cheverst, N. Davies, K. Mitchell, and A. Friday. Experiences of developing and deploying a contextaware tourist guide: The GUIDE project. In Proc. ACM/IEEE MobiCom), pages 20-31, Aug. 2000.

[8] Spatial Datasets. Website at http://dias.cti.gr/ ytheod/research/datasets/spatial.html.

[9] V. Gaede and O. Günther. Multidimensional access methods. ACM Computing Surveys, 30(2):170-231, Jun. 1998.

[10] A. Guttman. R-trees: A dynamic index structure for spatial searching. In Proc. ACM SIGMOD, pages 47$54,1984$.

[11] S. E. Hambrusch, C.-M. Liu, W. G. Aref, and S. Prabhakar. Query processing in broadcasted spatial index trees. In Proc. SSTD, pages 502-521, Jul. 2001.
[12] Q. L. Hu, W.-C. Lee, and D. L. Lee. Power conservative multi-attribute queries on data broadcast. In Proc. IEEE ICDE, pages 157-166, Feb. 2000.

[13] Q. L. Hu, W.-C. Lee, and D. L. Lee. A hybrid index technique for power efficient data broadcast. J. Distributed and Parallel Databases (DPDB), 9(2):151177, Mar. 2001.

[14] T. Imielinski, S. Viswanathan, and B. R. Badrinath. Power efficiency filtering of data on air. In Proc. EDBT, pages 245-258, Mar. 1994.

[15] T. Imielinski, S. Viswanathan, and B. R. Badrinath. Data on air - organization and access. IEEE TKDE, 9(3), May-June 1997.

[16] D. G. Kirkpatrick. Optimal search in planar subdivisions. SIAM J. on Computing, 15(2):28-35, 1983.

[17] R. Kravets and P. Krishnan. Power management techniques for mobile communication. In Proc. ACM/IEEE MobiCom, pages 157-168, Oct. 1998.

[18] W.-C. Lee and D. L. Lee. Using signature techniques for information filtering in wireless and mobile environments. J. Distributed and Parallel Databases (DPDB), 4(3):205-227, Jul. 1996.

[19] B. C. Ooi, R. Sacks-Davis, and K. J. Mcdonell. Spatial indexing in binary decomposition and spatial bounding. Information Systems, 16(2):211-237, 1991.

[20] Q. Ren and M. H. Dunham. Using semantic caching to manage location dependent data in mobile computing. In Proc. ACM/IEEE MobiCom, pages 210-221, Aug. 2000.

[21] T. Sellis, N. Roussopoulos, and C. Faloutsos. The $\mathrm{R}^{+}$tree: A dynamic index for multi-dimensional objects. In Proc. VLDB, pages 507-518, 1987.

[22] J. Xu, D. L. Lee, and B. Li. On bandwidth allocation for data dissemination in cellular mobile networks. ACM/Kluwer J. Wireless Networks (WINET), to appear, 2002.

[23] J. Xu, X. Tang, and D. L. Lee. Performance analysis of location-dependent cache invalidation schemes for mobile environments. IEEE TKDE, to appear, 2002.

[24] J. Xu, B. Zheng, W.-C. Lee, and D. L. Lee. The D-tree: A new index structure for querying locationdependent data. Technical Report, Dept. of Computer Science, Hong Kong Univ. of Science and Technology, Mar. 2002. 